\title{
Low-Dimensional Magnetic Systems
}

\author{
Roberto Zivieri, ${ }^{1}$ Giancarlo Consolo, ${ }^{2}$ Eduardo Martinez, ${ }^{3}$ and Johan $\AA$ Ǎkerman ${ }^{4}$ \\ ${ }^{1}$ Department of Physics, University of Ferrara, 44122 Ferrara, Italy \\ ${ }^{2}$ Department of Sciences for Engineering and Architecture, University of Messina, 98166 Messina, Italy \\ ${ }^{3}$ Department of Applied Physics, University of Salamanca, 37008 Salamanca, Spain \\ ${ }^{4}$ Department of Physics, University of Gothenburg, 41296 Gothenburg, Sweden \\ Correspondence should be addressed to Roberto Zivieri, zivieri@fe.infn.it
}

Received 2 August 2012; Accepted 2 August 2012

Copyright ( 2012 Roberto Zivieri et al. This is an open access article distributed under the Creative Commons Attribution License, which permits unrestricted use, distribution, and reproduction in any medium, provided the original work is properly cited.

The interest in the nanoscale properties of low-dimensional magnetic systems has grown exponentially during the last decades and has attracted the attention of both experimentalists and theorists. The state of the art of these investigations has indeed allowed to give valuable insights into the underlying physics of complex magnetization dynamics driven by magnetic fields, electric currents and thermal effects. At the same time, such studies have found, in relatively short times, several applications at industrial level in the field of spintronics and magnonics as magnetic memories, microwave oscillators, modulators, sensors, logic gates, diodes and transistors.

The goal of this special issue is to offer a variety of recent developments on this topic by gathering contributions arising from several specialists in the field of nanomagnetism. The strength of this issue lies indeed on its "variety": the properties of these systems are, in fact, investigated from the viewpoint of physicists, engineers and mathematicians. Also, the issue encloses studies carried out at both mesoscopic and atomic scales, as well as results of both theoretical approaches (analytical, numerical and, in some cases, even "hybrid") and experimental observations.

The covered topics range from the micromagnetic modeling of domain wall motion, dynamics of vortex structures, phase-locking phenomena in spintronic oscillators, experimental techniques for realizing heterostructures based on magnon-induced spin transfer torque, band structure and exchange field in the Landau-Lifshitz equation for magnonic crystals, gap and gapless structures in fractional quantum Hall effect, semiclassical description of anisotropic magnets and classical critical behaviour of Heisenberg ferromagnets. More specifically, within the subject dealing with domain walls, for example, the structure of complex cross-tie/vortex wall structures in soft films has been studied in detail by using micromagnetic simulations whereas the influence of the Rashba spin-orbit coupling on the current-induced dynamics has been investigated analytically. Regarding the exchange interaction governing the dynamics in magnonic crystals, a full analytical calculation of the exchange field acting on spin-wave dynamics from the microscopic Heisenberg model has been performed. Attention has been also devoted to the study of thermodynamics in the case of classical planar ferromagnets close to the zero-temperature critical point.

Two reviews are also included in this special issue. The first one deals with two hybrid micromagnetic tools, based on Hamiltonian and Lagrangian approaches, to model the spin-dynamics in laterally confined magnetic systems. The second one is mostly devoted to the micromagnetic analysis of static and dynamic properties of magnetic domain walls in materials exhibiting perpendicular anisotropy.

\section{Acknowledgments}

This issue is the result of generous contributions to this new journal from our colleagues. We are sincerely grateful to all of them for all their efforts. With papers by leading experts in the field of micro- and nanomagnetism, we hope that this special issue will form a timely, open-access resource for experts and novice alike.

Roberto Zivieri Giancarlo Consolo Eduardo Martinez Johan Akerman 

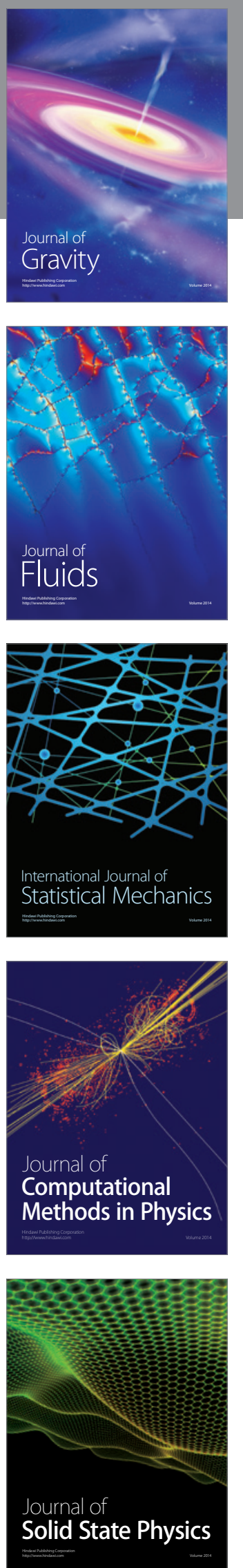

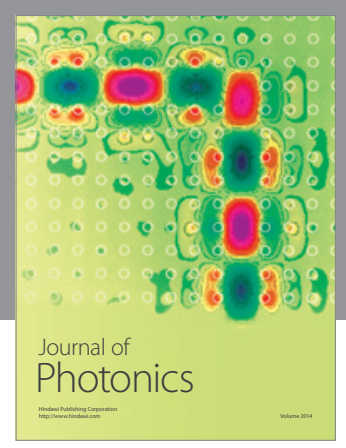

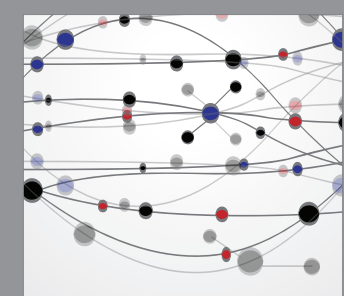

The Scientific World Journal
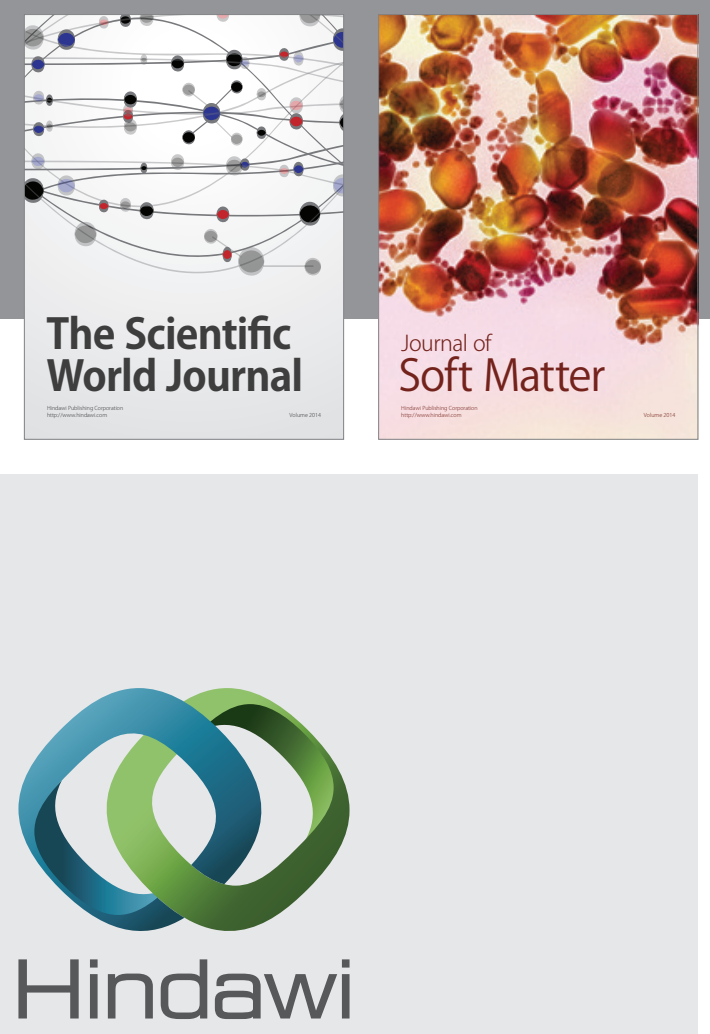

Submit your manuscripts at

http://www.hindawi.com
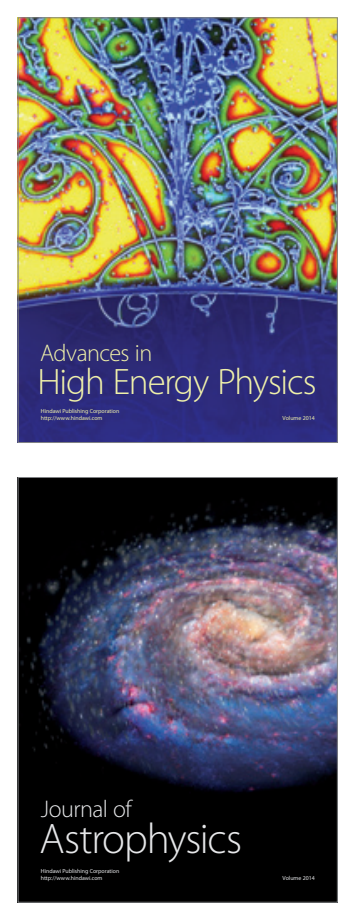
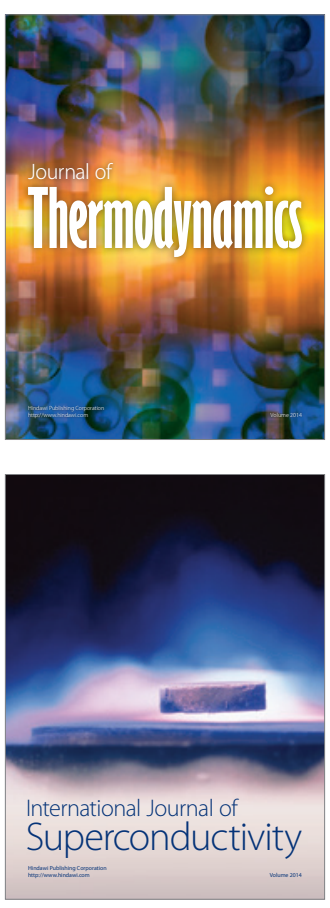
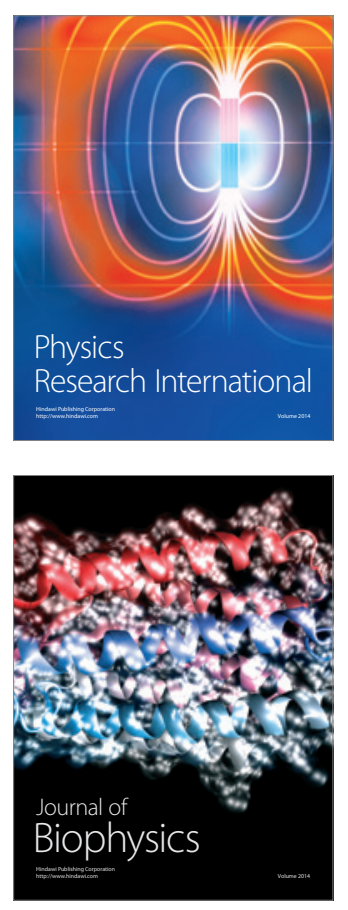
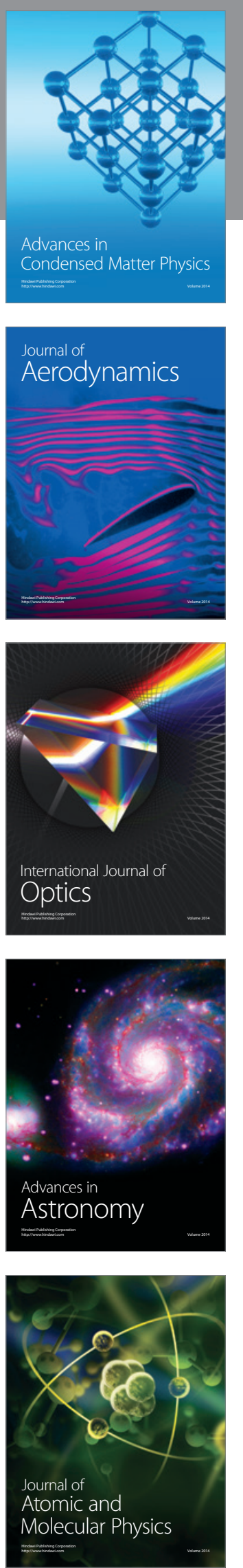